\title{
Effects of operating parameters and fluid properties on the efficiency of a new vacuum evaporation method
}

\author{
Johannes Rösti ${ }^{1, a}$, Dieter Baldinger ${ }^{2}$, Daniel Pulver ${ }^{3}$, and Heinrich Feichtinger ${ }^{4}$ \\ ${ }^{1}$ Agroscope, Route de Duiller 50, CP. 1012, 1260 Nyon, Switzerland \\ 2 ELVAmac SA, Route de Préverenges 10, 1026 Denges, Switzerland \\ 3 Agroscope, Schloss 1, Postfach, 8820 Wädenswil, Switzerland \\ ${ }^{4}$ hfc Consulting GmbH, Im Bad 1, 8132 Hinteregg, Switzerland
}

\begin{abstract}
A new process for vacuum evaporation was developed where evaporation takes place near the inner surface of a vortex as produced by a rotor submerged in the liquid. Contrary to the state of the art the new process does not need a vacuum vessel but the rotating liquid creates a geometrically stable low pressure void surrounded by a vortex stabilized by the equilibrium between centrifugal forces and the pressure difference. First tests with water and sugar solutions at concentrations similar to wine must showed evaporation rates in the upper range of thin-film evaporators. A test series was conducted to study the effect of the variation of process parameters. The heating power and thus the fluid temperature has the most important influence on the vaporisation rate. A second test series using sucrose solution of different concentration comes to the conclusion that this method is suitable for aqueous solutions but the vapour production rate drops significantly with increased sugar content using the current rotor design. The simplicity of the construction and the process handling make this new method a promising development for the wine production.
\end{abstract}

\section{Introduction}

Separation processes are a fundamental tool in process engineering. There is a wide range of methods of thermal, mechanical, chemical and biological methods for the separation of solid, liquid and gaseous mixtures. Extraction of a component of a mixture, concentration and cleaning are important operations in most industrial chains treating liquids of any kind. Important exponents are distillation, concentration and filtration in the food industry, treatment of wastewater, production of tap water, fractional distillation in the petroleum industry and the extraction of solvent in a chemical process.

This work focuses on the vaporisation of a liquid component as used in the food industry for the concentration of juice and wine and the distillation of fermented mash. Thermal processes such as evaporation and membrane separation are widely used in this branch $[1,2]$. There are specific requirements for many products since organic material can be affected by the treatment with high temperature, contact with oxygen and can contain solid particles.

A new vaporisation technique under reduced pressure for the concentration and separation of mixtures containing components with different boiling point has been developed [3]. After building a prototype and testing the new concept, this work explores the performance of

a Corresponding author: johannes.roesti@agroscope. admin. ch the system depending on the operation parameters and describes the underlying phenomena.

The main technical innovation of the new process is the generation of a reduced pressure zone using the centrifugal forces induced by a submerged rotor (Fig. 1). Rotating fast enough, the pressure in the centre of the rotor can be reduced sufficiently to reach the vapour pressure of the surrounding liquid or one of its components. Because of the dynamically generated pressure gradient, there is no need for a vacuum chamber nor a pumping system for the exchange of liquid between the low-pressure zone and the surrounding reservoir nor the usually required seals and valves.

For the prototype a hollow, tangential working rotor with radial blades or channels was used to generate the pressure gradient. Plates at the upper and lower end of the rotor terminate the rotating zone to prevent the elaboration of a secondary axial flow in the centre towards the lowpressure zone (Fig. 2). To improve the mixing of the fluid and prevent local concentration, it is possible to add an axial flow component inside the rotor (which is still restricted at the end plates). Stationary baffles around the rotor or a close-fitting tank wall limit the region of the rotating fluid. Without such a limitation, a vortex can be formed so that ambient air is sucked in because of the lowered level of the liquid surface. If the pressure gradient reaches the vapour pressure, a cylindrical vapour zone will be formed inside of the rotor. The so generated vapour can be extracted through an external condenser using a vacuum 


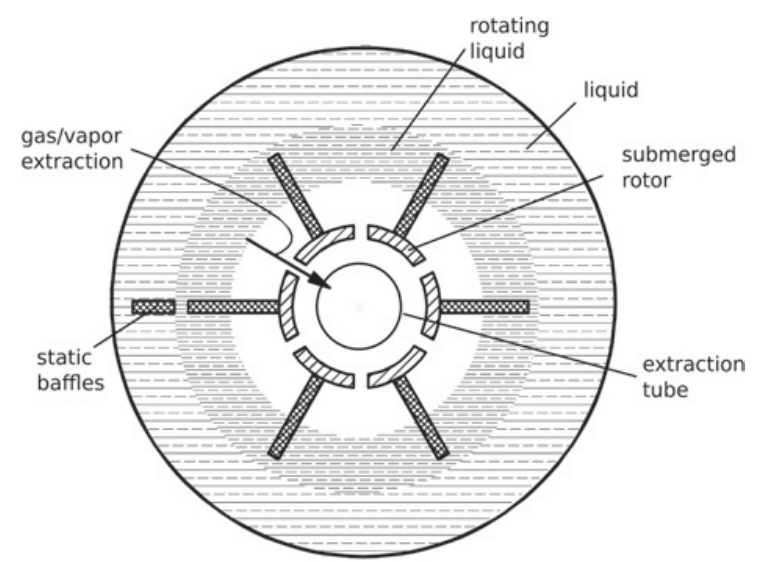

Figure 1. Main principle of the vaporisation method in a sectional view of the rotor (see also Fig. 2). Source [3].

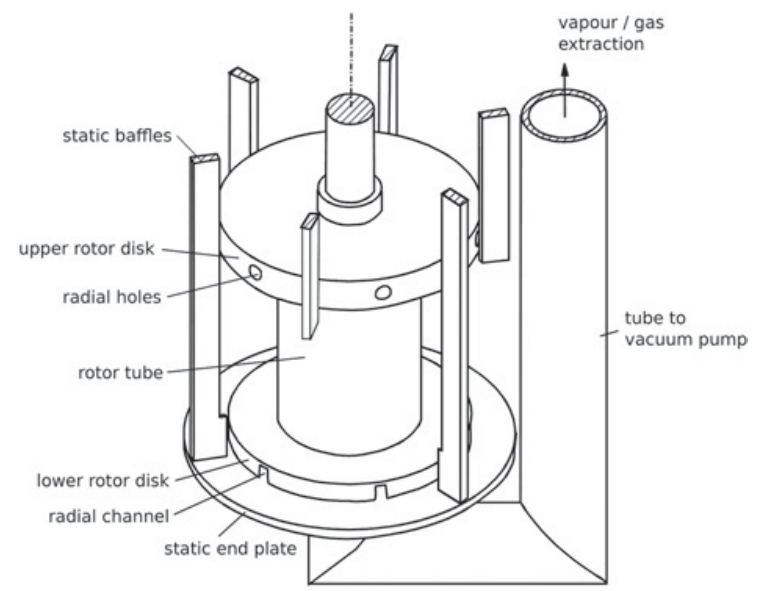

Figure 2. Implementation of the presented method using a rotor consisting of two disks with radial channels and a connecting tube in between. Source [3].

pump. The induced energy by the mechanical power input and an additional heating system have to compensate the energy dissipation of the vaporisation to keep the system running at a constant temperature.

First trials with the prototype already indicated the underlying effects causing the efficient vapour production. A short path between the liquid outside the rotor and the low-pressure zone in the centre enable a very fast exchange and bring the fluid entering the flashing zone suddenly in a superheated state. Theoretical considerations expect an exceeding flow rate through the rotor, which is an important advantage since a local concentration can be avoided and the heat is mixed homogeneously in the fluid. In addition, tests with grape must proofed the purity of the produced distillate, which is an indication of the strong centrifugal forces in the rotor preventing any liquid drop to reach the central exhaust tube.

Despite the various experiments conducted with the prototype, there are still many open questions about the underlying phenomena driving this process. The sensitivity of the vaporisation rate to the operating parameters have to be studied systematically to be able to control the process in every situation. The influence of the fluid properties is a further unknown, which has to be explored in detail in order to evaluate the possible applications of this process.

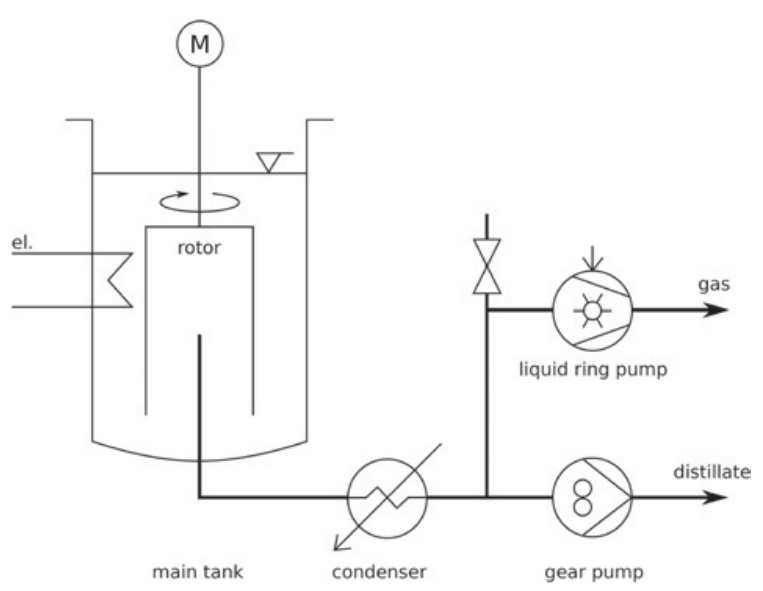

Figure 3. Plant schema of the prototype. Motor (M), electrical heating (el).

These questions are also of interest for the upscaling of the machine since the industrial applications require a higher vaporisation rate than it could be achieved with the existing machine.

The behaviour of the prototype was analysed using two test series. The first one was designed to test the influence of several operation parameters on the distillation rate of water while the second test series studied the vaporization of sucrose solution with different concentration.

\section{Materials and methods}

The new evaporation principle has been realized in a prototype built by hfc Consulting and Agroscope (Fig. 3). A support structure containing the motorisation and rotor was mounted on top of a $100 \mathrm{~L}$ tank so that the rotor was submerged in the liquid. The rotor was made of an aluminium tube with a diameter of $145 \mathrm{~mm}$ and a disk with a wider diameter at each end (Fig. 2). An electrical motor of $3.5 \mathrm{~kW}$ to drive the rotor was directly connected to the rotor shaft using a coupling without any gearbox. An additional speed sensor together with an inverter allowed controlling of the rotor speed with a resolution of $1 \mathrm{rpm}$. To compensate the heat losses due to vaporisation and losses to the environment, six electrical submerged heaters with a power of $1 \mathrm{~kW}$ were used. An external condenser, gear pump for the extraction of the distillate and vacuum pump were connected to the vapour zone using a pipe leading to the rotor from below (Fig. 3). The counter flow condenser used to cool down the water vapour in front of the vacuum pump was run with cool tap water of $12^{\circ} \mathrm{C}$. The small gear pump connected to the same condenser was used to pump the produced distillate to ambient pressure. The liquid ring pump was used for the extraction of noncondensable gas and increase the pressure gradient in the rotor centre. It worked with water as sealing medium and was therefore limited to the vapour pressure of the used cooling water. The instrumentation of the machine comprised temperature sensors (Type K) inside and outside the rotor, a pressure sensor $(0 \mathrm{mbar}$ to $200 \mathrm{mbar}$ with an accuracy of $0.5 \%$ ) and a data acquisition system (Legacy NI CompactDAQ, NI cDAQ-9172) with an input module for thermocouples and voltage sensing. LabVIEW working 
with a sampling rate of $2 \mathrm{~Hz}$ was used for on-line data visualisation and storage. A simple weighting scale with an accuracy of $1 \mathrm{~g}$ was used for the measurement of the distillation output in a certain time range. The stored data of the four temperature elements and the pressure sensor were cut and filtered in the desired range, where the process ran in a stable mode. A MATLAB GUI helped visually selecting the timespan for the used data points. Since the process did not show fast variations, the signals were smoothed by zero-phase filtering using a low-pass butterworth filter of order 4 .

In the first test series three factors with three values each were chosen. To be able to examine the test space in a suitable time span, a Box-Behnken design was chosen to obtain a linear regression model. The experiment using the central value for each factor was repeated at least once every day during the test session to check the reproducibility of the obtained results. For each experiment, the machine was running until the process reached an equilibrium. Once the process was running stable, the distillation output was measured six times. To ensure similar running conditions for the process, the temperature of the cooling water was monitored periodically. The influence of the three factors was tested using a linear regression model. Assuming a Gaussian distribution of the measured values around their corresponding real value allowed standardising the measurement data to obtain a regression model, which allowed comparing the factors among themselves.

With the content of about $90 \mathrm{~L}$ in the used tank, the stored heat lead to only slow changes in the temperature while the vaporisation was running. Therefore, dynamic experiments could be performed allowing scanning a wider range of operating conditions. For the second test series the liquid was heated up to $40^{\circ} \mathrm{C}$ before launching the machine and then let cool down during the experiment. The sugar content of the liquid was reduced stepwise from $27^{\circ}$ Brix. In contrast to the first test series, these experiments were run under continuous cooling caused by the vaporisation. The mass flux of the produced distillate was measured permanently. The experiment was run until an equilibrium was reached where the cooling due to vaporisation and heat losses to the ambient were equal to the continuously added stirring power. The manually measured data of the distillate production rate and the automatically recorded temperature and pressure measurements had to be synchronized to obtain a consistent data set. The recorded measurements from the data acquisition system were treated with the same procedure described above. In addition to the instrumentation used for the first test session, a refractometer was used for the analysis of the sugar content in the machine tank and the water quality of the distillate. The scale indicating the density in ${ }^{\circ}$ Brix allowed directly determining the amount of sugar in the liquid.

\section{Results}

The first parameter tested was the rotor speed since it is the main control parameter of the machine. Three different speed values were chosen to analyse the effect of different vapour cavity sizes and the resulting liquid flow in the rotor. At $1448 \mathrm{rpm}$ a small vapour zone was expected, with the gas/liquid surface $1 \mathrm{~cm}$ inside the rotor wall resulting in a thick liquid layer inside the rotor. At $1486 \mathrm{rpm}$ the gas/liquid zone was expected to fall close to the inner rotor wall resulting in a thin liquid layer inside the rotor. Finally, at $1542 \mathrm{rpm}$ an oversized vapour zone was created, where the liquid zone is theoretically pushed out of the inner part of the rotor. There is only liquid remaining in the upper and lower disk channels of the rotor.

The second test factor for this experimental design was the channel size of the lower rotor disk. It was expected, that the liquid flow through the rotor is an important condition for an efficient operation since a local increase of concentration or decrease of temperature would be adverse. Since preliminary tests have shown that the vaporization abruptly ceases when reducing the cross section of the channels by more than $90 \%$, the inlets were chosen to reduce the channel size by $0 \%, 33 \%$ and $66 \%$ resulting in channel cross-sections of $165 \mathrm{~mm}^{2}, 120 \mathrm{~mm}^{2}$, and $75 \mathrm{~mm}^{2}$, respectively.

As third test factor, the heating power was varied using $2 \mathrm{~kW}, 4 \mathrm{~kW}, 6 \mathrm{~kW}$ in addition to the $1.3 \mathrm{~kW}$ heating power originating from the mechanical energy of the rotor movement.

The main effects of the three test factors were analysed with a multiple regression model. While the rotor speed and the channel size were both not very important to explain the variation of the distillation rate, the heating power had a big impact (Fig. 4). Even this simple model neglecting the interactions between the tested factors was able to fit the data well as the adjusted coefficient of determination was 0.96 .

Although the port diameter of the inlet channel in the lower rotor disk does not seem to have much impact on the vaporisation in the tested range, it has to be stated, that the process did not work anymore when decreasing the open channel area more than $90 \%$. This is still an indication that these holes are important to maintain the internal flow in the rotor. There seems to be a saturation effect so that once the flow is established, a further increase of the opening does not improve the process any more.

A similar trend could be observed for the rotor speed. Once the rotor is able to create a sufficient depression, the radius and the inner flow do not affect the efficiency much - whether the liquid zone in the rotor is assumed to build a thick cylinder, a thin film or does not even reach the inner part of the rotor wall.

A second test series using sucrose solution of different concentration $\left(0^{\circ}\right.$ Brix to $27^{\circ}$ Brix $)$ was examined at different temperatures $\left(22^{\circ} \mathrm{C}\right.$ to $\left.40^{\circ} \mathrm{C}\right)$. Sucrose solutions exhibit similar characteristics as fruit juice and must regarding the density and viscosity. The increasing content of sugar had an important influence on the vaporisation rate (Fig. 5). In the tested range up to $40^{\circ} \mathrm{C}$, the distillate production decreased with the addition of sugar and caused the vaporisation to cease at about $30^{\circ}$ Brix.

\section{Discussion}

The two series of experiments provided the required data to work out the important effects describing the new 

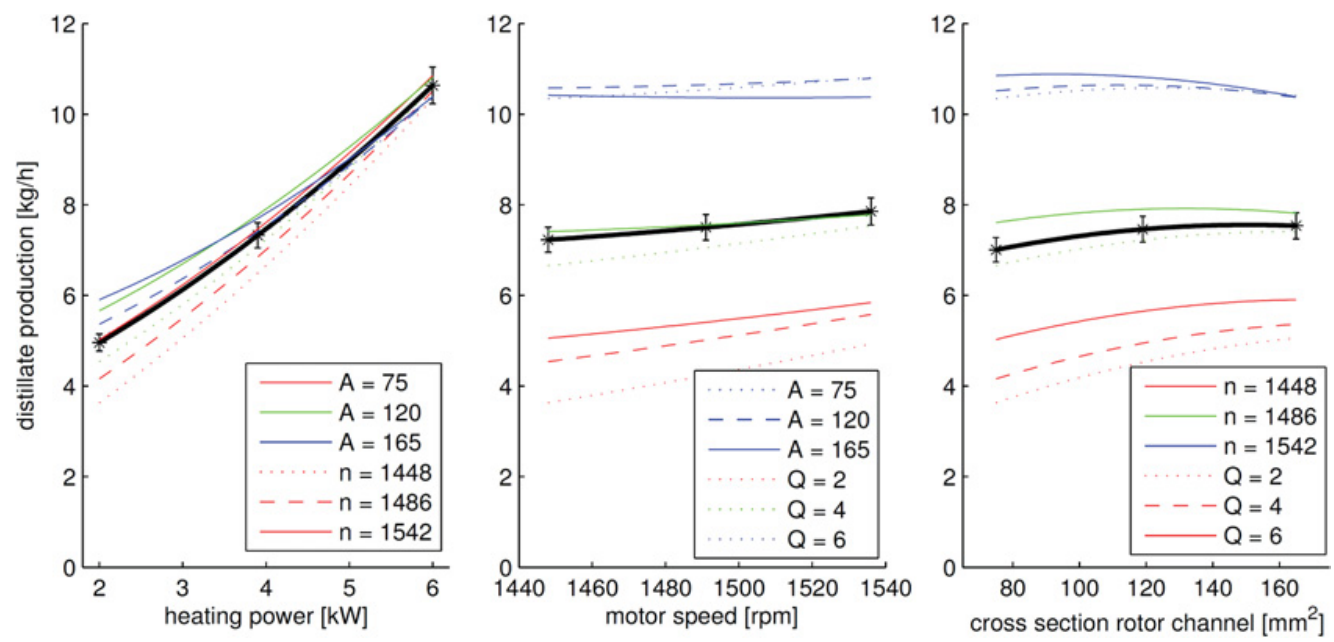

Figure 4. Effects of heating power (Q), motor speed (n) and cross section of rotor channel (A) on the distillate production rate. The black line indicates the central factor with error bars representing the measured standard deviation.

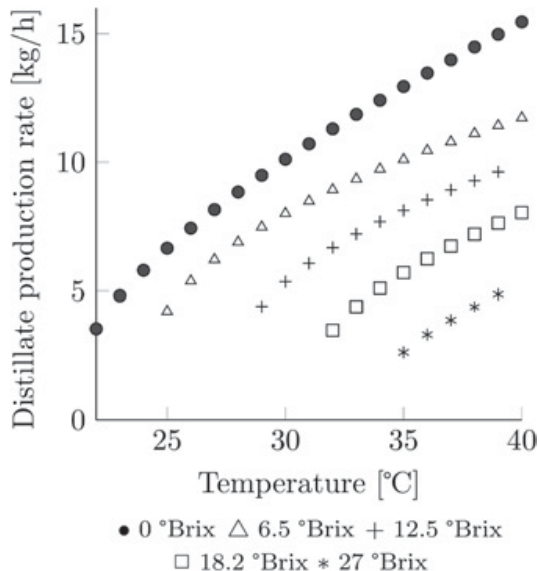

Figure 5. Effect of the fluid temperature and the sugar concentration on the distillate production rate.

vaporisation technique. Not only the level of superheat is important for the efficient flashing but also the absolute temperature of the fluid is a main factor. These two conditions can be easily controlled from the outside with the heating system and the vacuum pump. In contrast, the rotor speed does not have a major impact on the vaporisation rate. Although the circumference increases when the central cavity gets bigger at higher rotor speed, the vaporisation does not seem to increase with the same amount. A reasonable explanation is the reduction of width of the annular flashing zone because the pressure gradient drops faster at higher velocity. This effect compensates the higher centrifugal forces to a certain extent. In contrast to the limited enhancement by the rotor speed, this behaviour makes the process easy to control since the motor has not been able to provide a very precise speed. To increase the volume of the flashing zone anyway, a bigger but slower rotor could be used which also allows to scale up the machine. Once the rotor design is fixed, the system can be controlled by the temperature (or heating power) and inner pressure only. The maximum temperature of the fluid is determined by the specific applications in most cases. The remaining parameter is the power of the vacuum pump, which mainly influences the superheat of the flashing fluid. If a liquid ring pump is used as it is installed in the current prototype, the minimum pressure is defined by the temperature of the available cooling water.

While the vaporisation of water runs very efficiently, the distillate production drops significantly with the increasing amount of sugar and already ceases before reaching a concentration of $50 \%$ of the solubility limit of sugar. A feasible explanation for this drastic decrease is the retarded formation of bubbles due to the increased surface tension. The reduced bubble formation suspends a main advantage of this method since the bubbling and fast flashing liquid in the rotor centre is an important precondition for the efficient vapour production. To reduce this issue, a solution could be the artificial enhancement of the bubble content by the injection of additional small bubbles of air or inert gas in or around the flashing zone. The generated depression and the strong shear forces next to the edge of the rotor provide the ideal environment for such an installation.

The use of relatively cheap and simple accessible thermal power below $50{ }^{\circ} \mathrm{C}$ is an advantage of this system compared to alternatives such as reverse osmosis where all the required energy has to be provided in the form of electricity or high pressure. The simplicity of the construction and the process handling make this new method a promising development for the wine production.

\section{References}

[1] P. Cuénat, C.-A. Brégy, E. Zufferey, Revue suisse Vitic. Arboric. Hortic., 30, 307 (1998)

[2] J.-L. Berger, J. Int. Sci. Vigne Vin, 32 (1998)

[3] H. Feichtinger, WO 2013113131 (2013) 